\title{
Cultura escrita e Historia de la cultura
}

\author{
Literacy and the Cultural History
}

\section{AITANA MARTOS, AGUSTín VIVAS}

Universidad de Extremadura

España

Resumen. Leer y escribir son parte de una praxis social, de modo que pueden describirse como construcciones sociales fruto de las circunstancias y de los entornos culturales de cada época. La historia de la cultura se ha configurado como una disciplina que trata de describir estos procesos a partir de una metodología sistemática. Este artículo revisa los hitos principales en esta evolución científica y los autores que los han protagonizado.

Palabras clave: cultura escrita; historia de la cultura; cultura letrada; campo; literacidad.

\begin{abstract}
Reading and writing are a part of a social praxis so can be described as social constructs born of the circumstances and cultural environments from each time. History of Culture is configured as a discipline which aims to describe those processes from a systematic methodology. This article revises the main events in that scientific evolution and the authors who took part in it.
\end{abstract}

Keywords: written culture; history of culture; learned culture; literacy. 


\section{Los conceptos de "Cultura Escrita" y de "Historia de la cultura"}

Es preciso indagar el concepto más amplio de Cultura Escrita, en todas su dimensión histórica, partiendo del análisis de los primeros conceptos de Alfabetización y Paleografía que tanto se vinculan a él en sus orígenes, hasta llegar a la actual visión multidisciplinaria de la Cultura Escrita, abierta a enfoques tan diversos como los que suscitan los cambios históricos en la definición del lector y las prácticas de lectura ${ }^{1}$; los contextos de alfabetización; la construcción de la textualidad, los usos sociales de la lengua escrita, las relaciones entre la oralidad y la escritura, los distintos sistemas gráficos de representación y notación; la repercusión de las nuevas tecnologías en la Cultura Escrita; los procesos de difusión, recepción y apropiación del texto, etc. Lo más relevante, a nuestro juicio, es la focalización actual de la historia de la lectura entendida como práctica social y cultural.

En efecto, la Historia de la Cultura Escrita está indisolublemente ligada a la Historia de la Alfabetización y de la Paleografía. Para Sáez y Castillo Gómez (1994), la Paleografía es indispensable para cualquier elaboración científica de la Cultura Escrita. Como afirmara A. Petrucci ${ }^{2}$, si bien es posible una Paleografía sin Historia de la Cultura Escrita, ésta no se puede construir al margen de la primera. Desde esa óptica, la Paleografía ya no es sólo y simplemente la ciencia que estudia las escrituras antiguas, sino más bien una renovada disciplina que se plantea el estudio global de la historia de los usos y prácticas del escrito. Por lo tanto es la historia de los signos gráficos, pero también de la función y extensión social de éstos.

Es también la historia del libro y la lectura, así como de las formas de producción y conservación de la «memoria del saber» ${ }^{3}$. En cierto sentido podríamos decir que la Paleografía es una disciplina cuyo método y utillaje resulta necesario para una comprensión más enriquecedora y profunda del significado histórico-social de la

\footnotetext{
${ }^{1}$ Según las diferencias socio-culturales, están íntimamente relacionadas con la forma de lectura y transmisión de cultura, cf. CHARTIER, R. (2000) El Orden De Los Libros. Lectores, autores, bibliotecas en Europa entre los siglos XIV y XVIII. Madrid: Gedisa.

${ }^{2}$ En estos términos se expresó A. PETRUCCI en la conferencia que pronunció en Madrid el 28 de junio de 1991 dentro del curso Documentos y escrituras medievales en la Península Ibérica: Estado de la cuestión, celebrado en el Ministerio de Cultura.

${ }^{3}$ La memoria del sapere: forme di conservazione e strutture organizzative, a cura di Pietro Rossi, Bari, Laterza, 1990
} 
producción escrita, de lo que Virgilio de Toulouse llamó el «alma de la escritura» ${ }^{4}$. Por supuesto, sin renunciar al diálogo científico con otras disciplinas, tan necesario como productivo.

En consecuencia, esta reflexión sobre el quehacer paleográfico se plantea sin perder de vista el referente metodológico de la interdisciplinariedad, al que expresamente alude Antonio Viñao (1992) cuando reflexiona sobre el presente y futuro de la historia de la alfabetización. Así pues, cuando hablamos de Historia de la Cultura Escrita nos referimos a cualquiera de sus aspectos, como alfabetización, analfabetismo, historia del libro, de la edición, de las prácticas de lectura y escritura, etc.

Por su parte, la denominada Cultural History o nueva historia cultural se ha desarrollado y ha logrado su mayor apogeo en los medios académicos angloamericanos durante las dos últimas décadas. De modo paralelo a la relevancia alcanzada por la historia social en la historiografía contemporánea desde los años 60 , la versión socio-cultural de la misma ha conducido a la redefinición de la historia intelectual como una historia social de las ideas o mentalidades. Los trabajos de los historiadores producidos en los últimos treinta años han contribuido a cuestionar la visión reduccionista de la historia de la cultura como mero producto de las transformaciones socio-económicas. La convicción de que la cultura juega un papel decisivo como factor de cambio social es uno de los principales fundamentos de la nueva historia cultural.

Nos hallamos en un período de confusión motivado por la caída de unos paradigmas teóricos y el surgimiento de otros nuevos. En el estudio de la cultura, de por sí un concepto extremadamente complejo, han participado diferentes disciplinas académicas (antropología, historia social, lingüística, sociología y los estudios literarios, entre otras). Los estudios culturales se han constituido en un campo de estudio propio e interdisciplinar durante las tres últimas décadas, inicialmente en Gran Bretaña y, más recientemente, en Estados Unidos y otros países.

\footnotetext{
${ }^{4}$ RUIZ, E. (1992). Hacia una semiología de la escritura. Madrid: Pirámide.
} 
En los años 60 el interés de la historiografía por lo social fue promovido por la influencia de dos paradigmas interpretativos dominantes: el marxismo y la escuela de los Anales francesa. Es bien sabido que esta escuela promocionó el uso de métodos de análisis seriales, funcionales y estructurales para la comprensión de la sociedad como un todo integrado y orgánico. La primera generación de los fundadores de la escuela de los Anales (Marc Bloch y Lucien Febvre), oponiéndose a la historia positivista, abrieron el camino a una historia de los sistemas de creencias, valores y representaciones de un grupo social y de una época. Febvre sugirió la existencia en una época dada de una "estructura de pensamientos" determinada por la evolución socio-económica que promueve construcciones intelectuales y producciones artísticas. Ciertos "outils" mentales (palabras, símbolos, conceptos, etc.) sirven al pensamiento y los grupos sociales los utilizan de diferentes maneras. Ambos medievalistas desarrollaron la noción de que el pensamiento de los individuos y su particular expresión de los valores y creencias reposa sobre unos sistemas establecidos de pensamiento, "mentalidades" que difieren de una época a otra (Febvre, 1962).

La crítica de la historia de las mentalidades desarrollada a partir de la escuela de los Anales es la expresión más aparente de lo que en el mundo académico angloamericano se ha configurado como una nueva historia cultural, así como de las características del denominado Nuevo Historicismo.

Los trabajos de Michael Foucault, Philippe Ariés y Norbert Elias han contribuido significativamente a sentar los fundamentos de esta crítica. El más antiguo es The Civilizing Process de Norbert Elias (1939) que no fue realmente muy conocido hasta su traducción al francés en 1973. En su visión del "proceso civilizador", Elias (1978) atribuye los cambios producidos en la historia europea al poder social de los valores establecidos por las élites. Para Ariés (1992) el desarrollo de la idea de los ciclos de la vida individual preparó el camino a la idea de progreso a través de la civilización. En el caso de Foucault (2004) el concepto de "poder-conocimiento" es clave en su teoría, la cual, ha sido considerada como una respuesta alternativa al marxismo y a la escuela de los Anales al mismo tiempo. Si bien es bastante generalizada la opinión de que su mayor contribución ha sido metodológica y temática más que teórica. 
La influencia que la antropología y la crítica literaria han ejercido en la configuración de una nueva historia cultural es inseparable del interés que la semiótica ha suscitado en los historiadores. Los sistemas de signos y el significado que se esconde detrás de ellos es uno de sus mayores focos de atención. El término semiótica, acuñado en América por el filósofo Charles S. Peirce y el término semiología, acuñado por Ferdinand de Saussurre, se usan hoy indistintamente aunque ha habido intentos de diferenciarlos. Entre los años 60 y 80 la semiótica ha desarrollado un conjunto de conceptos y una terminología que han inundado diversos campos de estudio. Saussurre defendió la configuración de la semiótica como una parte de las ciencias sociales y no como una mera técnica de aproximación al estudio de los signos. En esta orientación social de la semiótica ha sido influyente la teoría de Mikhail Bakhtin (1986) sobre la equivalencia entre el dominio de los signos y el de la ideología. Lévi-Strauss, así como Robert Scholes más tarde, utilizan el paradigma lingüístico en sus estudios de antropología cultural.

Uno de los temas que ha producido mayor número de estudios ha sido la definición de la cultura popular, tradicionalmente interpretada como la cultura de los no alfabetizados o menos instruidos y en oposición a la cultura de la élite o minoría de instruidos. El interés de los historiadores por la cultura popular pasó de la periferia al centro a partir de los años 60 gracias a trabajos como los de Serla Ginzburg en Italia, Robert Mandrou y Natalie Davis en Francia, Keith Thomas en Inglaterra y Julio Caro Baroja en España. Aunque hubo anteriormente una larga tradición de folkloristas que se remonta a los siglos XVIII y XIX entre los que destacan la escuela alemana con Wolfgang Brückner, Gerhard Heilfurth y Otto Clemen y la finlandesa con Anti Aarne y Kaarle Krohn.

El descubrimiento de la cultura popular fue parte del movimiento en busca del primitivismo del siglo XVIII, en lo cual lo antiguo, lo lejano y lo popular se equiparaban. Fue también una reacción en contra de la razón ilustrada y su negación de la tradición. La mayoría de los estudios sobre cultura popular medieval se centran en la Baja Edad Media y en la temprana Edad Moderna. Así, Mullett (1987), acentúa la continuidad de rasgos culturales medievales en los siglos XVI y XVII en relación con las formas de protesta y control social. La cultura popular del siglo XVIII en Inglaterra 
cuenta con los estudios clásicos de Hoggart (1959) para la clase trabajadora y Stone (1964) en relación con el acceso a la educación desde 1560 a 1640, además del más reciente con nuevas aproximaciones de Vicent (1989) que cubre hasta principios del presente siglo.

\section{Corrientes y Estudios sobre de la Cultura Escrita.}

Hablar de las relaciones históricas entre la sociedad y la escritura o la lectura, los dos componentes que definen el alfabetismo, es hacerlo de lo que en el ámbito anglosajón se ha dado en llamar literacy y en Italia -al no encontrar una sola palabra con la que traducir el término- alfabetismo e cultura scritta. Esta noción fue definida por Attilio Bartoli Langeli (1978: 28) -uno de sus principales impulsores junto a Armando Petrucci- como el estudio de todos los usos activos y pasivos de la escritura, la extensión cuantitativa de los mismos, el análisis de los contenidos culturales e ideológicos transmitidos y difundidos mediante la escritura o el grado de prestigio social alcanzado en cada momento por las personas alfabetizadas y sobre todo por quienes se dedican profesionalmente a la escritura.

La inquietud historiográfica por el estudio de la escritura desde una perspectiva social se consolidó en la década de los sesenta, con aportaciones científicas de antropólogos e historiadores, preocupados por las transformaciones culturales acarreadas con la introducción de la escritura y la cuantificación de la extensión social de las capacidades de leer y escribir en el Antiguo Régimen. Paralelamente, dieron sus primeros frutos las semillas plantadas a lo largo de la primera mitad de siglo respecto al estudio de la escritura en su contexto histórico y social, alumbrándose entonces la renovación del concepto paleográfico y su orientación hacia el alfabetismo y la Cultura Escrita.

Dichos antecedentes se pueden rastrear en la obra de Scipione Maffei, Istoria diplomatica che serve d'introduzione all'arte critica (Mantua, 1927). En ella se define la Paleografía como "historia de la escritura", si bien su insuficiente teorización hizo que no se tomara demasiado en serio. 
Poco después, en el marco de la Europa de entreguerras y contemporáneamente al nacimiento de Annales, dichas ideas empezaron a tener mayor consistencia. Primero fue el alemán Ludwig Traube, destacado representante de la historische Paläographie, es decir del estudio de la escritura en cuanto actividad humana y, por lo tanto, como un aspecto no secundario de la historia de la cultura. Y luego, sin olvidar las aportaciones de otros autores ${ }^{5}$, se produjo el alumbramiento de las teorías, ya aludidas, de István Hajnal ${ }^{6}$.

Las reflexiones del paleógrafo húngaro -cuya obra ha sido calificada por Armando Petrucci (1993) como verdadera y propia anticipación en el desarrollo de una historia de la escritura que privilegie el aspecto de las relaciones entre este instrumento de expresión y la sociedad contemporánea-, reflejan un discurso más elaborado de la teoría e historia de la escritura indisociable del contexto social que la produce. En los años sesenta se hacen más nítidas las perspectivas abiertas en las décadas anteriores y terminan dando origen a una rica corriente de investigación que tiene en la escritura y el alfabetismo dos polos de referencia.

El campo histórico centrado en el estudio del analfabetismo y la alfabetización es uno de los que evidencia con más claridad los cambios experimentados por la historiografía contemporánea en los últimos cuarenta años ${ }^{7}$. En estas cuatro décadas, la

\footnotetext{
${ }^{5}$ Luigi Schiaparelli, Jean Mallon y la escuela franco-belga, Giulio Batelli o Giorgio Cencetti. El análisis de la contribución al desarrollo científico de la Paleografía por unos y otros autores puede examinarse con la lectura de diversos trabajos como el artículo de A. Pratessi «Uno sguardo al passato per affacciarsi al futuro», en Un secolo di paleografia e diplomatica (1887-1986). Per il centenario dell'Istituto di paleografia dell'Università di Roma. Roma: 1988, pp. XIV-XXI y otros incluidos en esa misma obra.

${ }^{6}$ HAJNAL, István. L'Enseignement de l'écriture... Op. Cit.

7 Seguimos en esta exposición de manera predominante las líneas de trabajo elaboradas por Antonio Viñao, Petrucci), Francisco Gimeno Blay y Antonio Castillo Gómez. Sobre esta evolución en los estudios sobre el mundo del analfabetismo y la alfabetización y las últimas tendencias historiográficas, véase: VIÑAO FRAGO, A. (1984). Del analfabetismo a la alfabetización. Análisis de una mutación antropológica e historiográfica. Historia de la Educación, 3, 151-189 y 4 (1985), 209-226; «Historia de la alfabetización versus Historia del pensamiento, o sea, de la mente humana», Homenaje al profesor Alesandre Sanvisens. Barcelona: Universidad de Barcelona, 1989, pp. 375-387 (este artículo se publicó también en la Revista de Educación, n. 288 (1989), pp. 35-44); «Alfabetización y alfabetizaciones», en ESCOLANO BENITO, A. (dir.), Leer y escribir en España. Doscientos años de alfabetización, Salamanca, Fundación Germán Sánchez Ruipérez, Madrid, Pirámide, 1993, pp. 385-410. PETRUCCI, A. (1989). Storia della scrittura e della società», Alfabetismo e cultura scritta, nuova serie, 2, 47-63; Scrivere per gli altri, Scrittura e Civiltà, 13, 475-487; Storia della Scrittura e storia della società, Anuario de Estudios Medievales, 21, 309-322; La scrittura descritta, Scrittura e Civiltà, 15, 5-20; Scrittura come invenzione, scrittura come espressione. Estudis Castellonecs, 6, 1093-1100. Asimismo véase, GIMENO BLAY, F. (1986). Las llamadas ciencias auxiliares de la historia: ¿errónea interpretación?
} 
historia de la alfabetización ha atravesado por tres fases o etapas. En un primer momento, se restringía a una mera descripción del fenómeno. En una segunda fase, la renovación que estaba experimentando la historiografía en general alcanzó también a los estudios sobre alfabetización que se realizaron desde nuevas disciplinas -historia de la educación, historia de la escritura, sociología, psicología...- y que incorporaron nuevas variables y centros de interés -implicaciones ideológicas, culturales, económicas, etc.-. La tercera y, por ahora, última etapa ha ampliado un poco más los límites de los estudios históricos sobre alfabetización mediante la toma en consideración de elementos pertenecientes al mundo de la comunicación, del lenguaje y del pensamiento.

\subsection{Los estudios tradicionales}

Como algunos investigadores, entre ellos Stone y Schofield, han reconocido la alfabetización fue casi totalmente ignorada tradicionalmente por los estudios históricos.

Una rápida lectura de los índices de los trabajos de historia de la educación o incluso de historia social e historia económica anteriores a la década de los años sesenta del siglo veinte, muestra sólo de manera ocasional una breve referencia a la alfabetización. Este tema aparece, sin embargo, en algunos escasos trabajos que pueden clasificarse, según Harvey J. Graff (1981) en tres categorías principales: estudios de alfabetización de las élites o de grupos especiales, estudios en los que los niveles de la alfabetización de distintas poblaciones o subgrupos son deducidos indirectamente más que medidos directamente $\mathrm{y}$, por último, estudios en los que la alfabetización es periférica a otros temas examinados ${ }^{8}$.

Los primeros estudios sobre este fenómeno llevados a cabo en España se caracterizaban por centrar su interés en el analfabetismo -casi exclusivamente decimonónico-, realizar una descripción de la distribución y evolución del analfabetismo por sexos, edad, provincias, etc. y acompañarla de una enumeración de sus causas. La mayoría de los autores que pueden ser encuadrados dentro de este

(consideraciones sobre el método de investigación en Paleografía). Zaragoza. CASTILLO GÓMEZ, A. (1995). De la Paleografía a la historia. De las prácticas del escribir», en BARROS, C. (ed.). Historia a Debate, T. II, Santiago de Compostela, pp. 261-271; «Del signo negado al signo virtual. Cambios y permanencias en la historia social de la Cultura Escrita», Signo. Revista de Historia de la Cultura Escrita, 6, 113-143. CASTILLO GÓMEZ, A. y SÁEZ C. (1994). Paleografía versus alfabetización. Reflexiones sobre historia social de la Cultura Escrita», Signo. Revista de Historia de la Cultura Escrita, 1, 133-168.

${ }^{8}$ GRAFF, H. J. Literacy in History. Op. Cit., p. 12. 
epígrafe consideraba únicamente un modo de alfabetización: el que se desarrollaba a través del sistema escolar formal. Estos primeros estudios sobre alfabetización y analfabetismo estaban comprendidos dentro de la denominada historia de la educación.

Harvey J. Graff, uno de los principales historiadores de la alfabetización, ha distinguido dos generaciones desde entonces hasta mediados de los ochenta, cuando ciertos cambios hacían presagiar el nacimiento de una tercera ${ }^{9}$. Sin embargo el balance que este autor realiza no tiene en cuenta la bibliografía paleográfica, cuyos primeros frutos en relación al alfabetismo se dieron paralelamente al desarrollo historiográfico que él traza.

\subsection{Hitos generacionales}

Con el antecedente próximo de las obras de M. Fleury y P. Valmary (1957), el nacimiento de la historiografía de la alfabetización tiene lugar, según Graff, con la publicación de las primeras obras de Lawrence Stone (1964), Carlo Maria Cipolla (1969), Jack Goody (1963) o Roger S. Schofield (1968), en las que formulan la historia del alfabetismo como finalidad científica, establecen las primeras series cronológicas de la difusión del alfabetismo, identifican las fuentes necesarias para su estudio, principalmente las de tipo cuantitativo, inician el camino de los estudios comparativos a partir de la variable de la firma y se plantean, a veces de forma especulativa, los factores más decisivos y sus consecuencias cognitivas y sociales. Pero también nacen entonces algunas interpretaciones un tanto unívocas de los procesos de la alfabetización que luego se han revelado insuficientes e inadecuadas, como la identificación entre la alfabetización y el desarrollo socioeconómico o político, el llamado « mito de la alfabetización»».

Se completa esta primera etapa con la coetánea aparición de importantes estudios sobre la historia social del libro y la lectura, según se desprende de algunas publicaciones colectivas y las obras de Henri-Jean Martin, continuando los caminos abiertos a finales de los cincuenta por el propio Martin y Lucien Febvre.

\footnotetext{
${ }^{9}$ GRAFF, H. (1986). The History of Literacy: Toward the Third Generation. Interchange, Special Anniversary Issue, Illuminating Education: The Uses of Science, History, and Philosophy in Educational Thought, 17(2), 122-134.
} 
A partir de finales de la década de los años sesenta, se produjo un vuelco en estos estudios y se acentuaron causas, conexiones o implicaciones ideológico-culturales más complejas; se desplazó la atención del analfabetismo hacia el proceso de alfabetización, sus agentes y sus modos de actuación.

A la primera generación sucede otra de tipo más globalizante y especulativo ${ }^{10}$ cuyas aportaciones se resumen en el mayor aprovechamiento de las series cuantitativas, la complejidad de los procesos de la alfabetización, la importancia atribuida a la contextualización de los mismos, el reconocimiento de los factores ideológico-culturales y su influencia en la conformación de diversos modelos históricos de alfabetización, la investigación de los usos del alfabetismo, tanto en sus plasmaciones prácticas -escritura y lectura- como en sus efectos psicológicos, así como la profundización en el discurso teórico y metodológico, señalando las contradicciones y dificultades de la historia de la alfabetización, la importancia de los estudios comparativos y las limitaciones del cuantitativismo.

Además todo ello se vio favorecido por el desarrollo paralelo de la nueva historia del libro y de la lectura, emparentada con Annales, que la sitúa entre los «nouveaux objects» de su Faire de l'histoire $(1974)^{11}$. Los principales círculos eran en francés y el anglosajón ${ }^{12}$.

\subsection{Renovación historiográfica de la disciplina: paso de la Alfabetización a la}

\section{Cultura Escrita.}

Bajo esta nueva interpretación del proceso alfabetizador que implicaba factores ideológicos, políticos y religiosos surgieron una serie de investigaciones con nuevos

\footnotetext{
${ }^{10}$ A la que pertenecen nombres como Egil Johansson, Kennet A. Lockridge, Furet, Sachs y Ozouf, Michael T. Clanchy, David Cressy, L. Soltow y E. Stevens, Rab Houston y Graff, más las nuevas obras de Goody y Schofield.

${ }^{11}$ CHARTIER, R. y ROCHE, D. (1979).El libro. Un cambio de perspectiva. En LE GOFF, J. y NORA, P. (dir.) Hacer la historia, III: Objetos nuevos. Barcelona: Laia, p. 115-137.

${ }^{12}$ Son muchos los historiadores franceses del libro, la imprenta o la lectura, algunos también con incursiones en la historia de la educación, que podríamos mencionar, por lo que nos limitaremos a señalar los más importantes: Martiu, Chartier, Compere y Dominique Juliá, Hébrard, Jacques Queniart, R. Mandrou, R. Muchebled o Natalie Zemon Davis. Fuera del ámbito francés, pero en estricta relación con dicha temática, está la innovadora y controvertida investigación de E. Einsenstein sobre los efectos de la imprenta en el pensamiento y las diversas publicaciones de P. Burke sobre la cultura de las clases populares.
} 
enfoques, cuestiones e interrogantes, que intentaron ilustrar diversos momentos del proceso alfabetizador, especialmente europeo ${ }^{13}$.

La profundización en el conocimiento de la multiplicidad de agentes relacionados de alguna manera con la difusión de la alfabetización implicaba, a su vez, un análisis de la relación entre alfabetización y crecimiento económico que se alejase de la simplicidad de explicaciones monocausales que simplemente atribuían a la alfabetización los mejores resultados económicos de un país. Los nuevos análisis que se produjeron a partir de los años sesenta permitieron introducir nuevas variables en los estudios sobre alfabetización.

El cambio historiográfico trajo como consecuencia una inversión total del objeto de estudio, que pasó del analfabetismo a la "alfabetización como proceso" 14 . Éste quizá haya sido el cambio más evidente e importante, ya que permitió descubrir toda la riqueza, variedad y complejidad de lo que venimos denominando proceso de alfabetización que, en realidad, encierra otros muchos en su seno: diversos modelos nacionales o regionales de alfabetización, distintos procesos de alfabetización: el de lectura y el de escritura y, por último, confluencia de distintas disciplinas en su estudio historia del libro, historia económica, sociología...--

Los nuevos métodos de análisis abrieron el camino a investigaciones sobre distintos modelos históricos de alfabetización y a las diferencias que también existían en su interior. Constituida la historia de la alfabetización, bajo sus múltiples y convergentes enfoques, en una dinámica corriente historiográfica, a finales de los setenta y principios de los ochenta alcanzó una mayor proyección académica fuera de los tradicionales círculos anglosajón y francés.

Mediados los setenta, la historia del libro y la lectura entra a formar parte de los «nuevos objetos» de estudio, y en este sentido es especialmente relevante la

\footnotetext{
13 A modo de ejemplo pueden ser citados: CRESSY, D. (1980). Literacy and the Social Order. Cambridge: Cambridge University Press; CLANCHY, MT. (1993). From memory to written record, England 1066-1307. Oxford: Cambridge, Blackwell, o FURET, F. y OZOUF, J. (1977). Lire et écrire. L'alphabetisation des Français de Calvin à Jules Ferry. Paris.

${ }^{14}$ VIÑAO FRAGO, A. (1985) (1985). Del analfabetismo a la alfabetización. Análisis de una mutación antropológica e historiográfica. Historia de la Educación, 3, 151-189; y 4.
} 
aportación de Roger Chartier, quien en sus investigaciones sobre las prácticas de producción, circulación y lectura de libros en las sociedades del Antiguo Régimen, establece el vínculo entre la historia del libro y la historia de la lectura al reconstruir aquélla como las formas contrastadas con que lectores diferentes aprehendían, manejaban y se apropiaban de los textos contenidos en un libro.

Superado el enfoque estadístico de las primeras publicaciones sobre el alfabetismo, a principios de los ochenta, era posible una especialización que atendiera más los aspectos antropológicos y sociológicos del uso de los sistemas de comunicación gráfica ${ }^{15}$. Lo que había nacido como historia del alfabetismo se estaba convirtiendo progresivamente en una investigación sobre aspectos tales como el proceso de la alfabetización (o alfabetizaciones), sus agentes, modos y vías, la difusión de la mentalidad letrada, las funciones y usos de la escritura y la lectura, los contextos y prácticas de difusión y recepción de lo escrito, las relaciones entre la cultura oral y la Cultura Escrita...

En Italia su difusión supuso la continuación respecto a los pilares establecidos en los años sesenta por Armando Petrucci. Un hito destacado, en esta línea, fue la celebración del seminario Alfabetismo e cultura scritta nella storia della societá italiana en la ciudad de Perugia, convocado por Attilio Bartoli Lanbeli y Armando Petrucci. Especialistas de diversas disciplinas se reunieron para contrastar sus respectivas experiencias y conocimientos sobre un tema que aún no había tenido demasiado eco en la historiografía italiana: la escritura y la lectura, el alfabetismo y la alfabetización o la producción y disfrute de la cultura a través de lo escrito, analizados en el ámbito de la historia social, según explican sus organizadores ${ }^{16}$.

Con la organización de este congreso, sus promotores se habían propuesto tres objetivos que el mismo Attilio Bartoli Langeli desarrolla en su Intervento di apertura. En primer lugar, introducir los estudios de Paleografía en un circuito de intereses y motivaciones históricas más amplio y significativo, contribuyendo a ampliar la

\footnotetext{
${ }^{15}$ CARDONA, G. (1999). Antropología de la escritura. Barcelona: Gedisa.

${ }^{16}$ Presentazione, in Alfabetismo e cultura scritta nella storia della società italiana. Atti del Seminario tenutosi a Perugia il 29-30 marzo 1977, Perugia.
} 
metodología, los instrumentos y el campo de investigación de la disciplina. En segundo lugar, iniciar un discurso común sobre la escritura y sobre sus implicaciones socioculturales, poniendo en común las propuestas de método y los diversos puntos de vista desde los que el fenómeno había sido o podía ser estudiado. En tercer y último lugar, hacer tomar conciencia de que el estudio de la escritura no podía continuar en manos de disciplinas especializadas, incomunicadas entre ellas, sino que debía ser objeto de una valoración histórica compleja: es decir, proponer a los historiadores un campo de trabajo cuyas potencialidades el estado de los estudios, en ese momento, no había mostrado.

2.4 Nuevas perspectivas para el estudio de la historia de la lectura y escritura: el enfoque sociocultural.

La renovación que ha protagonizado la historiografía de la lectura con las sucesivas aportaciones de la Escuela de los Annales de Chartier, la historia de la vida privada, las llamadas "escrituras populares" (Lyons 2007) y otros estudios culturales referidos a estas materias, se ha visto completada desde otros paradigmas. A estas aproximaciones clásicas se les ha unido un nuevo tipo de estudios socioculturales que ponen el énfasis en las interacciones comunicativas, en los entornos y en sus organizaciones sociales, las instituciones que "patrocinan" la alfabetización, etc.

La orientación que adoptan hoy buena parte de las investigaciones sobre literacidad es sociocultural, por oposición a otros enfoques que ponen el acento en los aspectos lingüísticos (enfoque lingüístico) o psicológicos (enfoque psicolingüístico). Dentro de esta corriente sociocultural, podemos situar líneas de investigación más específicas, como las siguientes. Veamos algunos de sus principios:

1. Cualquier escrito está "situado" en un contexto concreto.

2. El lector no está solo. Vivimos en comunidad: interpretación plural.

3. Cada comunidad tiene sus formas particulares de leer y escribir. El uso del lenguaje varía con el espacio y el tiempo. 
4. El significado se origina en la comunidad. Las palabras solo transmiten rasgos muy esquemáticos, que deben completarse en cada comunidad.

5. La literacidad tiene origen social.

6. La literacidad es un fenómeno cultural e históricamente situado.

\subsubsection{El paradigma de los Nuevos Estudios de Literacidad}

Un ámbito especialmente interesante son los llamados Nuevos estudios de literacidad, practicado fundamentalmente por autores anglosajones. Las investigaciones sobre lectura basados en los trabajos de la corriente de los Nuevos estudios sobre literacidad Street, 1984, 1995; Heath, 1983; Gee, 1992, 1996; Barton, 1994, Barton y Hamilton, 2000; Hamilton, 2000) han subrayado que la lectura no es sólo una competencia individual ni centrada exclusivamente en la escuela sino una práctica social que cobra sentido en una comunidad dada, es decir, que se sitúa en un contexto que la conforma.

Echando mano de una perspectiva etnográfica, entienden la lectura y la escritura como prácticas sociales, en las que las personas utilizan los textos, situados sociohistóricamente, dentro de contextos particulares, para desarrollar funciones concretas, en el seno de instituciones establecidas, con unas relaciones de poder determinadas. Entre otras, cabe citar algunas fuentes que pertenecen a esta corriente:

1. Estudios etnográficos clásicos: Brice-heath, Scollon y Scollon, Scribner y Cole. Gee (1990); Barton et al. (2000).

2. Estudios hispanos: Zavala (2002), Ames (2002).

Así pues, para dar cuenta de las nuevas prácticas de lectura y de escritura que surgen en la sociedad contemporánea, aparecieron una serie de estudios luego catalogados como New Literacy Studies, que conceptualizan la lectura como una práctica social contextualizada (aptitud...para ser usada en todos los ámbitos, con miras a conseguir objetivos personales y extender los conocimientos y capacidades). Se diferencia bastante, por tanto, de la visión considerada tradicional, que no destaca apenas la percepción de los hechos y prácticas "letradas" dentro de ciertos contextos y 
pautas sociales. De modo que sería el uso y el contexto social el que articularía de forma plena el sentido, valores, o la finalidad de la comunicación en cuestión.

Además, esta visión considera la comunicación de la lectura y la escritura en un sentido integrador dando paso a otras literacías posibles. Así pues, en esta visión, la alfabetización es ante todo un conjunto de prácticas sociales. Los estudiosos de esta corriente (Brian Street, James Paul Gee, Allan Luke, Colin Lankshear, Michele Knobel...) defienden que la literacidad no es una competencia autónoma sino herramientas indisociables de la comunidad y el contexto en el que se aplican.

Todos estos nuevos enfoques nos llevan a considerar que, en lugar de una alfabetización o literacía canónica, debamos hablar hoy de una literacía plural o múltiple que según el grupo canadiense de la Universidad de Otawa (2006), se ramificaría en tres "literacías" básicas:

a) Alfabetización escolar

b) Alfabetización social o comunitaria

c) Alfabetización personal (ámbito familiar)

No cabe duda de que cada una de estas instancias (escuela, comunidad -en el sentido de barrio, ciudad...) alfabetiza al individuo de una manera diferente, y que lo que los "eventos" y prácticas letradas de un hogar, un taller o un aula divergen considerablemente. Contrariamente a los estudios tradicionales, no hay una alfabetización ideal estándar, sino una alfabetización plural, y la pedagogía debe tener en cuenta estos tres ejes. Aprendemos a usar un texto participando en los contextos en que se usa (Cassany, 2006), de forma que la adquisición de la literacidad no es una simple tarea académica que pueda desligarse del entorno familiar y ciudadano.

Igualmente, conforme a la teoría de las inteligencias múltiples, se ha hablado también de otros tipos de literacías o alfabetizaciones, referidas a otras realidades, como la comprensión de los números, el espacio, etc. Lo cierto es que, en el contexto la cultura pos-tipográfica y digital en que nos hallamos, en una época de globalización y de mezcla (relacionada con la estética llamada posmoderna), la consideración de "letrado", para el siglo XXI, no es quien simplemente maneja mecánicamente un 
ordenador o códigos de la lengua escrita o se expresa en dos idiomas: el "letrado" es quien sabe cambiar de "registro" según las demandas de la situación, y por tanto sabe usar lo mismo textos expresivos que argumentativos, y sabe "zapear" la lectura y la escritura desde una nota manuscrita a la lectura de una noticia, una enciclopedia o una página web.

Veamos los conceptos centrales que se proponen desde este paradigma de estudio, según Cassany (2006):

\section{Práctica letrada.}

2. Hecho o evento letrado (communicative event).

3. Texto escrito.

4. Institución u organización social. Disciplina epistémica, ámbito o grupo humano.

5. Identidades y roles de autor y lector.

6. Valores y representaciones de la lectura.

7. Género discursivo: función, estructura, estilo, retórica.

8. Historia particular de la práctica letrada.

Se trata de describir las prácticas de lectura que sean observables en distintos ámbitos. Hay que subrayar la diferencia entre las prácticas letradas vernáculas (que preferimos llamar "domésticas", como el uso de recetas, diarios, notas...) de las prácticas letradas dominantes (textos formales, informes, exámenes...). En las prácticas "domésticas" hay una apropiación de la escritura hacia fines muy particulares. La escuela y la sociedad privilegia unas en detrimento de otras, por ejemplo, se presta poca atención a lo que produce el lector en interacción con un texto, o a los apuntes, materiales de estudio, escritos expresivos...

Sin embargo, hay aprendizajes sociales no escolares, es decir, la comunidad es un agente alfabetizador importante, y además estos dominios o contextos son en realidad ámbitos permeables, que se solapan a menudo. Veamos al respecto una lista de 
eventos o prácticas letradas que pueden formar parte de la vida de un escolar, según las edades, los usos y los entornos.

Hay que trazar, pues, una tipología de prácticas y eventos letrados, según sea el ámbito de la escuela, la familia, los amigos... En cada ámbito se da un número limitado de eventos letrados, por ejemplo en la escuela se hallan limitados a las adquisiciones escolares, mientras en casa hay numerosas prácticas letradas cotidianas que nada tienen que ver con la lectura o la escritura académicas, como es leer/escribir un recado, la nota de la compra... Del mismo modo, en casa estas prácticas están vinculadas a tareas concretas y a prácticas de ocio, mientras que en la lectura escolar y en la "escritura académica" se practica otras estrategias y experiencias. Por último, en la casa hay más escritura que lectura, mientras que en el colegio es habitual que haya poca producción escrita y más comprensión lectora.

Por tanto, es una cuestión de ensanchar o acortar el abanico de posibilidades, lo que Goody ha llamado littératie restreinte o elargie, es decir, cultura escrita de uso restringido o bien expandido, extendido. Los eventos letrados son las actividades observables en las que la lectura y/o la escritura se desarrollan, pero en el trasfondo operan las prácticas surgidas de la comunidad que dan valor a la cultura escrita en su seno.

Barton y Hamilton (2000) describen los eventos de literacidad como las actividades observables en las que la lectura y/o la escritura se desarrollan. Estas actividades siempre están insertas en contextos sociales y emergen de prácticas de literacidad que definen formas culturales para utilizar el lenguaje escrito. Frente a los eventos letrados, las prácticas no son del todo observables, pues se dan también al interior de los individuos e incluyen valores, actitudes y creencias compartidas por grupos, fomentando así la dialéctica entre lo tangible y lo intangible, tan propia del mundo de la lectura y la escritura.

2.5 Los enfoques sociocomunicativos: la ecología de la comunicación y la definición de campo en relación con la cultura escrita (Bourdieu).

El sociólogo francés P. Bourdieu (1992) estableció como concepto central de su 
teoría el "campo", partiendo de la analogía de este concepto en física. Así, sostuvo que los campos sociales son espacios de juego históricamente constituidos con sus instituciones específicas y sus leyes de funcionamiento propias. Lo importante es que, como en el juego, subyacen una serie de reglas y que el sistema tiende al dinamismo, a actualizar distintas potencialidades, y que, dentro de este "juego", se confrontan poderes, capitales, etc.

En lo relativo al arte y la cultura, no hay un arte unívoco sino producciones legitimadas y aceptadas por los grupos hegemónicos o bien producciones de los grupos subalternos, en interacción con aquéllas. Así que por muy autónomo que parezca el objeto o el bien cultural de que se trate (la literatura, el patrimonio histórico...) no hay campo que sea

Objetivement indépendant de ce qui se joue à l'extérieur ; les conflits qui s'y déroulent ont une logique interne, mais le résultat des luttes (économiques, sociales, politiques) externes au champ pèsent fortement sur l'issue des rapports de force internes ; par exemple, les « hérétiques » du champ littéraire pourront trouver un appui dans l'émergence de nouvelles clientèles, liée à certaines transformations du système scolaire ${ }^{17}$.

El campo literario o cultural es el espacio resultante de todas estas mediaciones. Y, sobre todo, no es campo plácido o en armonía, sino dominado por los conceptos de rivalidad y competición:

[...] un espace structuré de positions, un réseau de relations objectives entre des agents ou des institutions qui s'interdéfinissent par la distribution inégale de ce capital spécifique, soit, dans le cas précis du champ littéraire, les salons, les maisons d'édition, les revues, les écoles, etc. L'inégale répartition de ce capital est au principe des luttes qui animent le champ (et qui, par ailleurs, contribuent toujours à son autonomie -de là le fait que le champ est toujours inséparablement le lieu et le produit des luttes entre les agents partageant la même activité). La seule relation qui soit, en définitive, structurante, est le rapport de domination.

17 Atelier de théorie littéraire : Définition $\mathrm{du}$ champ, en http://www.fabula.org/atelier.php?D\%26eacute\%3Bfinition_du_champ 
Les formes prises par les luttes au sein du champ littéraire - en tout cas depuis que s'est affirmée son autonomie - sont invariantes et en nombre limité, au premier rang desquelles on peut noter l'opposition entre orthodoxie et hétérodoxie (i.e. toute tentative de subversion qui oblige les dominants à affirmer l'orthodoxie et imposer la doxa). Les luttes ont pour objet l'appropriation d'un capital spécifique mais peuvent aussi avoir pour objet la redéfinition de ce capital; en ce cas, elles peuvent modifier profondément la configuration du champ en redistribuant radicalement le capital selon les nouvelles normes imposées ${ }^{18}$.

Aplicando estos principios a la descripción de la configuración de la cultura escrita como sistema dentro de una obra tan emblemática como el Quijote, A. Martos (2008) distingue con nitidez cuatro macrocampos o dominios, orientados pragmáticamente de forma distinta: la cultura de creación o de innovación; la cultura orientada a la instrucción-socialización; la cultura vinculada a la preservación-memoria y, finalmente, la cultura material, que trata de la producción, los soportes, etc.

Cada dominio tiene sus propios agentes semiótico-materiales, pero lo importante no es el artefacto cultural considerado aisladamente (por ejemplo, el libro), sino, como decimos, las "relaciones en juego", la "competencia" a través de las diversas prácticas que se pueden reconocer en la obra y que se hallan legitimadas dentro de la cultura hegemónica y/o en las culturas subalternas.

La teoría de campos de P. Bourdieu deriva, según Martínez (2008), en una especie de topología social, basada en la noción de un espacio no cuantitativo, donde lo que interesa son las relaciones de orden y de posición entre los elementos de las distintas partes del espacio o "campo de juego". Por tanto, no interesa la figura o posición aislada, el "individuo", lo "singular" sino la red de relaciones o principios relacionales y la configuración o construcción mental que seamos capaces de establecer.

Las ideas de Bourdieu y de Cassanova acerca de que dentro de un campo lo que hay son zonas en conflicto se puede ilustrar, a propósito de la lectura, con la descripción de Joaquín Aguirre, que él compendia sobre lo que podríamos denominar

18 Ibidem. 
"macrocampos" relacionados con el mundo de la cultura escrita, que estaría integrado por los siguientes sectores principales:

1. Creación,

2. Productivos-distributivos, consumidores

3. Educativos" (en sentido amplio).

4. Clasificadores.

En apariencia, el enfoque de esta división es puramente sincrónico y funcional, pero puede ser también histórico-descriptivo sí aplicamos a estos subsistemas principales que configuran el sistema de la lectura, las teorías de Bourdieu o la teoría de los polisistemas de Even Zohar.

De forma, pues, sincrónica, el sistema literario-cultural es entendido por J. Aguirre como el conjunto de prácticas y movimientos de circulación que rodean la producción, la distribución y el consumo de los textos en nuestra cultura, que conforman esas "zonas de conflicto", con ejes muy diversificados, que priman unas veces el individuo, el placer, el ingenio, la capacidad de emprender (incluyendo la heterodoxia), y otras veces, al contrario, lo colectivo, la socialización, la legitimación social, la ortodoxia; o bien el eje que describe la producción material, el beneficio, la conservación de los bienes, la inclusión/exclusión en una tradición, canon o repertorio.

Esta misma idea de dinamismo conflictivo es también expresada en los Nuevos Estudios de Literacidad. Según Barton y Hamilton (2000: 8), en la cultura escrita actual hay prácticas más dominantes, visibles e influyentes que otras, pero sólo por cuestiones relacionadas con el poder o la economía, no porque su naturaleza sea inferior. De este modo, las construcciones de las identidades culturales/lectoras (rol social) son manifestadas a través de diversas conductas comunicativas, que son las que moldean las prácticas letradas, y las que hacen que un estudiante llegue a primar los apuntes y manuales como artefactos básicos y casi únicos, orientados a un fin pragmático, y desposeídos habitualmente de toda la riqueza que caracteriza al mundo intelectual, la "polifonía de voces", y no el "pensamiento cristalizado y anónimo". A este respecto, A. 
Martos (2008) adaptó dicha idea de topología social o delimitación de campo, en forma de cuadrícula, para describir la cultura escrita en el Quijote:

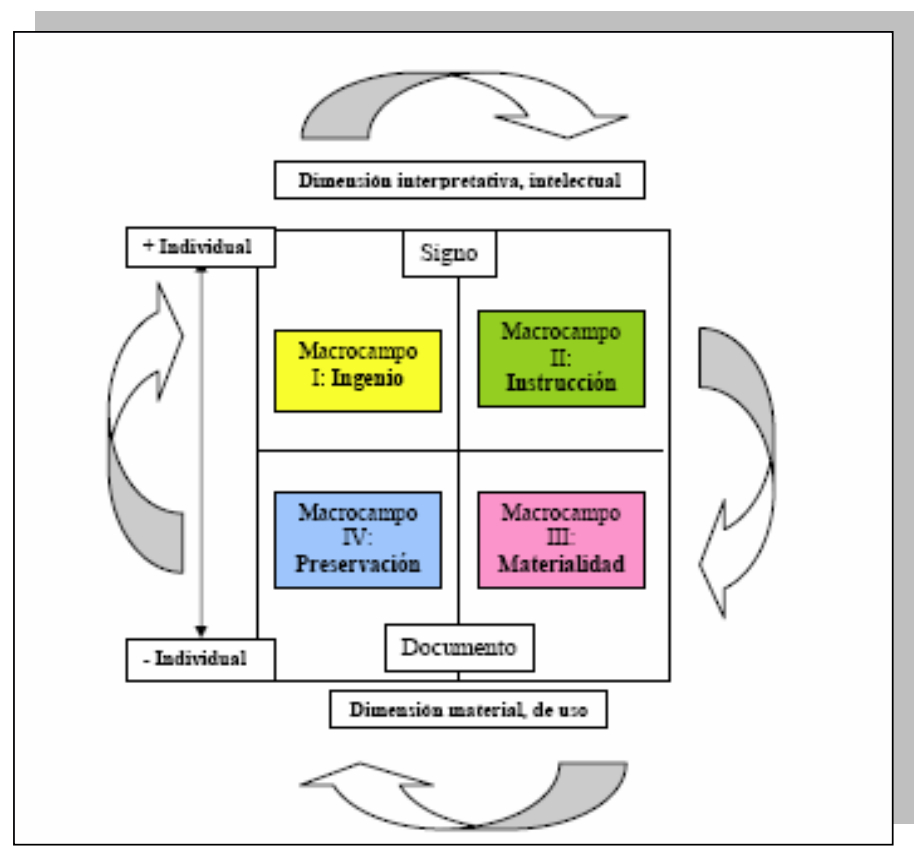

En este caso, distinguimos con nitidez cuatro macrocampos o dominios, orientados pragmáticamente de forma distinta: la cultura de creación o de innovación; la cultura orientada a la instrucción-socialización; la cultura vinculada a la preservaciónmemoria y, finalmente, la cultura material, que trata de la producción, los soportes, etc. Cada dominio tiene sus propios agentes semiótico-materiales, pero lo importante no es el artefacto cultural considerado aisladamente (por ejemplo, el libro), sino las prácticas contextualizadas y los elementos influyentes: disparidad de conexiones, heterogeneidad y multiplicidad.

Porque estos subsistemas están en interacción, incluso en "fricción", en medio de contextos culturales y políticos que tienden a instrumentalizar el conocimiento y el progreso científico. Por ejemplo, bajo muchas de las políticas más activas de editoriales y librerías de promoción de la lectura subyace un modelo de industrialización de la cultura, se trata ante todo de consumir o comprar más, de plegarse a las modas, al "instantaneísmo" tan propio de la red. Y eso casa mal con la cultura letrada y su constante apelación a los clásicos, estén de moda o promoción, o no. 
Es justo lo que pasa con la cultura letrada, Barton y Hamilton (2000: 8) aseguran que en la cultura escrita actual hay prácticas más dominantes, visibles e influyentes que otras, pero sólo por factores relacionados con el poder o la economía, lo mismo que ciertos cursos o máster parecen representar la cúspide del conocimiento, pero se vinculan a ciertas aspiraciones profesionales o de status. Ciertamente, el pensamiento reduccionista y binario (letrado/iletrado, sabio/ignorante, oral/escrito, literario/audiovisual...) tiende a disolverse en estos nuevos escenarios, e igual que ya están desacreditadas las reducciones escrito $=$ culto, oral=popular, $\sin$ duda el acercamiento a los nuevos "alfabetismos", dará lugar a una consideración más global y omnicomprensiva de la literatura, por ejemplo, la apertura de la misma hacia las textualidades electrónicas que se están produciendo.

En efecto, la cuadrícula de A. Martos es un esquema que visualiza las tesis de Chartier, quien defiende la síntesis entre la textualidad del libro y la materialidad del texto, o, dicho de otro modo, cómo la cultura material en torno al libro, de un lado, y los valores de cosmovisión, poética u originalidad, se dan como posibilidades reales dentro de este "campo de juego", que Cervantes lleva al extremo gracias a una calculada ambigüedad y a saber hacer converger continuamente la cultura letrada más refinada con el saber popular de raíz local. Por eso, el Quijote es un compendio o una especie de enciclopedia de la cultura escrita de la época, y, por lo mismo, una especie de máquina de plantear interrogantes y preguntas sobre todo lo que rodea a la cultura escrita, esto es, a la mente letrada en el sentido de Olson (1994), que usa la escritura para autocomplacerse en esos pensamientos e historias -fingidas o reales- que le llevan hacia el mundo interior, hacia la autoconciencia.

\section{La Cultura Letrada y la Cibercultura, nuevos horizontes. Hacia un concepto expandido e integrador de la Cultura Escrita}

En suma, resulta muy esclarecedora la idea de dinamismo y de confrontación aplicadas a los dominios o campos de la cultura, según las tesis explicadas de Bourdieu y cómo se trasladan a las mediaciones y conflictos de los distintos agentes. Por ejemplo, aplicando la lógica bajtiniana, la dialéctica del Quijote parece enfrentar en apariencia, la oralidad y 
la escritura, el caballero y el rústico, el hombre y la mujer... pero todas estas confrontaciones se pueden entender mejor a la luz del concepto de circularidad $^{19}$.

Estas "filtraciones" es lo que viene a estudiar los Nuevos Estudios de Literacidad cuando subraya que no sólo el centro académico es una comunidad alfabetizadora; la familia, los amigos o "pares” y, por supuesto, Internet están funcionando, en la práctica, a modo de culturas subalternas (Castillo Gómez, 2001b) que sin embargo son capaces de introducir sus "cuñas" en la cultura oficial, en las prácticas dominantes, y no nos referimos sólo a que se centre en contenidos distintos, alternativos, sino a aspectos más esenciales, a que se base en otros modos de lectura y escritura, que sí están socavando las estructuras clásicas más conocidas. Por citar solamente un caso, la habituación al hipertexto y su no-linealidad es una forma de antagonismo respecto a la lectura secuencial del libro impreso, que está marcando unas nuevas actitudes de lectura.

A este respecto, Olson subraya la interrelación escritura-conciencia, y sostiene que lo importante de la cultura escrita es que contribuye a que fijemos la atención o seamos capaces de repensar los propios pensamientos o representaciones, es decir, a que construyamos o elaboremos un pensamiento propio (en sintonía con las teorías expresadas por Havelock en "La musa aprende a escribir"). Así pues, el objeto de la cultura letrada no sería la erudición como tal, el conocimiento por el conocimiento, sino esta capacidad de repensar las cosas y de forjar con ello un mundo propio, una autoconciencia. Eso es lo más "revolucionario": que la escritura nos lleva a la conciencia y la reestructura (Ong, 1987), posibilitando una introspección, una interiorización de las representaciones y un distanciamiento entre las ideas y las palabras que las expresan, pasos esenciales del pensamiento crítico.

En este contexto, la "ciudad letrada" se parecería a una "ciudad rizomática", estratificada en múltiples niveles, y que respondería esta cultura de lo complejo. Los

\footnotetext{
${ }^{19}$ Este concepto permite reconocer un proceso de filtración de información en un doble sentido en sociedades jerarquizadas, donde los sectores que representan a la cultura caligráfica o letrada con la elaboración de sus discursos y teorías sistemáticas se posicionan para explicar la realidad, y a partir de esta dinámica de posicionamiento poder permear y moldear las prácticas de otros grupos sociales no letrados denominados culturas subalternas. Pero, de igual forma, estos grupos subalternos logran atravesar la cultura letrada o hegemónica con las prácticas discursivas que ellos elaboran, fundadas sobre el entramado dinámico de la oralidad, es decir, se va dando un flujo constante de permeabilidad o cruce de información desde ambas partes, desde lo letrado, y desde lo no letrado. (Tamara Garay, 2005), vid. http://casadeasterion.homestead.com/v6n22ginz.html
} 
principios rizomáticos ${ }^{20}$ no nos describen centros, jerarquías o linealidades, sino múltiples planos o "mesetas" dentro del "rizoma" planetario, que funcionan como zonas de distintas intensidad pero que establecen continuas conexiones entre ellas.

La ecología comunicativa que describiese el mapa mundial de la alfabetización y de la cultura escrita tendría este mismo aspecto rizomático de sistemas descentralizados, que conectaría línea dinámicas, flujos, entre múltiples subsistemas; que no tendría comienzo ni fin, porque, para las personas involucradas, siempre estaría en el medio. Estas "comunidades alfabetizadoras", ya se trata de centros de enseñanza, la familia, los grupos de iguales, etc. trazarían el diseño de este "mosaico" de la cultura global, el cual, como la propia red, no tiene centro sino rizomas, ramificaciones.

Con esta nueva visión se abre una percepción distinta, por ejemplo, una mayor aproximación a lo cotidiano o un mayor interés por las culturas locales, en sintonía con lo que postulan nuevas corrientes historiográficas, como la Microhistoria de Carlo Ginzburg. Por consiguiente, el énfasis ya no está en el conocimiento, la literatura o el saber en abstracto, sino en las personas y en sus prácticas contextualizadas, de ahí la importancia del enfoque perfomativo.

Dichas prácticas afloran de forma especial en contextos de emergencia, por ejemplo, la Universidad ha puesto en valor el tema de la alfabetización académica, igual que para las Bibliotecas es esencial la alfabetización informacional o que la red está impulsando un tipo de industria de entretenimiento que subsume gran parte de la herencia letrada (es el caso ya aludido de la ficción fantástica o juegos de aventuras, terror o fantasía y sus referentes "letrados"). Las distintas descripciones de estos nuevos escenarios han puesto de relieve las relaciones subyacente entre conocimiento, poder o status, y, al modo de la descripción cuasi etnográfica propuesta por los Nuevos Estudios de Literacidad, han desvelando que, tras cualquier evento letrado, subyacen unos participantes, un ámbito de encuentro, unos artefactos y una serie de actividades y rutinas, con manifestaciones tanto explícitas como implícitas.

\footnotetext{
${ }^{20}$ DELEUZE, G. \& GUATTARI, F. (1980). Capitalisme et Schizophrénie 2. Mille Plateaux. París: Minuit
} 
La difusión de la cultura letrada clásica, como modelo de organización de los estudios, el saber y las academias o disciplinas, no se apoya precisamente en el modelo “circular" e igualitario de Internet, sino más bien en un esquema vertical, jerárquico y hegemónico. Por eso la cibercultura, con las nuevas formas colaborativas y cooperativas en la producción de saberes, ataca este sistema dominios y de privatización del saber y fomentan la cultura de libre acceso.

\subsection{Las prácticas letradas electrónicas}

Internet desarrolla una dinámica participativa, donde aparte de los libros, autores o editoriales, lo que cobra auge son las "comunidades interpretativas", por tanto son estos “e-lectores" la fuente de los nuevos significados. Esto es, los nuevos lectores de la era digital, pegados a su PC, móviles y otros dispositivos, no sólo están contribuyendo a nuevas formas de cibercultura y de ciberliteratura, sino que contribuirán también a apropiarse de los clásicos “a su manera. En todo caso, estos clásicos no serán olvidados, como temen los que denostan la cultura digital, sino más bien "revisitados", actualizados conforme a los nuevos valores, preferencias y lenguajes, por ejemplo, se recurre a la multimodalidad, pues sin duda la imagen, el cine o los multimedia seguirán siendo una "pasarela" importante de acceso a la cultura literaria clásica.

Es difícil imaginar en el futuro un público más o menos homogéneo, como el que acude a ver una ópera o un concierto. Parece más probable que haya comunidades interpretativas estratificadas, esto es, colectivos de significación que tienen gustos distintos y que, internamente, comparten lecturas similares, conformando una subcultura susceptible de crear una "comunidad virtual" 21 , la cual organizará sus propias acciones, celebraciones y eventos. De alguna manera, es lo que ya está pasando con los aficionados al rol, la fantasía, la ciencia ficción o el terror.

La red será, pues, un inmenso laberinto donde el lector se hará intérprete de un supuesto "mapa del tesoro", esto es, de lo que le interesa y atrae, debiendo para ello trazar itinerarios, búsquedas o cartografías dentro de un territorio que desconoce. El mediador, el profesor, se enfrenta, pues, a cometidos nuevos: ayudar en estos tanteos o

\footnotetext{
21 "Virtual" en el sentido de alternativo, opuesto a lo habitual, no en el sentido de contrario a lo real.
} 
esbozos, acompañar al "extraviado"; interpretando así, las lecturas salvajes o caóticas a que se refiere Chartier formarán parte de forma inevitable de este nuevo lector digital. Además, estas comunidades ya no se forman, como en la cultura letrada clásica, a partir de ciertos niveles socioeconómicos; más que por afinidades económicas, el mundo de los fans o de estos nuevos lectores se nutre por afinidades de edad, géneros o cualquier otra seña de identidad compartida, tal como ocurre en el deporte de masas.

La teoría de la recepción lo expresa de forma clara: leer activamente es extraer el "potencial de sentido" de un texto, es pues, una tarea constructiva. Por tanto, lo importante no es la naturaleza del objeto en sí, que sea un libro o la voz de un poeta recitando poemas o cualquier otro soporte, lo que importan son las prácticas de lectura y escritura, así como los contextos y las interacciones que median en ellas.

La experimentación artística y la revolución tecnológica harán que nuevos artefactos culturales convivan con el artefacto cultural por excelencia, el libro. Como si se tratara de un paraguas, cada artefacto desplegará todas sus posibilidades y cambiará o transformará las relaciones, como ya está haciendo el e-book o la lectura on line, y todo ello se hará desde una construcción interpersonal, desde esta cultura de la convergencia y de la participación (Jenkins, 2008).

Debemos fomentar las ideas de Daniel Link al diferenciar entre la cultura audiovisual de masas posindustrial, es decir, los medios de comunicación masivos e internet; y al afirmar que hay una competencia por el tiempo libre entre la cibercultura y la cultura industrial, pero no entre la cultura letrada y la cibercultura que funcionan como aliadas. Ante el problema que planteaba Chartier sobre cómo pasar de una "lectura salvaje" -o caótica- a la cultura letrada, Daniel Link entreveía soluciones desde el momento en que Internet y la cultura letrada son más aliadas que ésta y la cultura industrial o audiovisual de los medios masivos (que sólo parecen ofrecer “entretenimiento anestésico").

Las vías de integración de ambas culturas es el familiarizar al ciudadano con las dos, esto es, fomentar prácticas culturales "anfibias" e incluso híbridas, eclécticas en todo caso, de forma que sea factible desplegar todos los eventos y artefactos culturales útiles y de interés; es algo que ya se viene produciendo en el ámbito de Internet, en net- 
art, la ciberliteratura y todas las formas de experimentación, en particular aquellas que, como hemos visto en sucesivos ejemplos, actualizan, reinterpretan y reconfiguran los contenidos y textos de la cultura letrada. Además, la oportunidad de educar en los nuevos alfabetismos y el esfuerzo por hacer estas integraciones de contenidos y tradiciones nos va a obligar a una consideración más global e interdisciplinar.

Las anteriores consideraciones deben llevarnos a la comprensión de la complejidad de la situación actual, donde no se puede definir la cultura escrita en términos simples o unívocos. De hecho, la dialéctica más elemental nos obliga a repensar el papel de la oralidad, la cultura impresa, la cultura electrónica y los nuevos escenarios culturales, porque estamos en un entorno donde se simultanean los públicos y personajes (i)letrados, los escenarios ágrafos y las "salas” o ámbitos letrados, según que haya o no presencia de la cultura letrada. Estamos, pues, en una cultura híbrida.

Lo que pudiéramos llamar una "cultura letrada holística" debe saber aproximar estas realidades y superar las preconcepciones más enraizadas, como la de que la cultura letrada es lo "antiguo", lo "clásico" o lo "erudito", y que se "encapsula" en cánones o normas centrales que irradian hacia la periferia, hacia los "iletrados". Hoy sabemos por las aportaciones de Chartier, Ong, Havelock, Olson y tantos otros que los mecanismos son complejos y que estamos ante una cultura "híbrida", pues hasta los más "frikis" de la cultura digital usan para sus historias, sabiéndolo o no, referentes de la cultura oral o impresa. El dialogismo bajtiniano supone saber reconocer el juego entre un valor y su contrario, algo que la Red practica, por ejemplo, al parodiar la cultura letrada o los "contenidos serios".

La nueva Cultura Letrada será, pues, un ámbito híbrido donde se pueda oír plenamente lo que Michael Oakeshott (2009) llama "la voz del aprendizaje liberal", a saber, un lugar donde confluyan voces, herencias y discursos radicalmente, diversos, pues para este autor la cultura no es un conjunto de creencias, de percepciones o de actitudes, o un cuerpo de conocimientos o canon, sino una variedad de lenguajes de comprensión mediados por distintos agentes semiótico-materiales.

En conjunto, la nueva cultura letrada, además de su estructura compleja, múltiple e híbrida, se configuraría a sí misma a través de prácticas cercanas a estos tres 
principios: dialogismo, performatividad y activismo ${ }^{22}$. En el primer principio, está claro que en una cultura hay una serie de voces diferentes, y obtener acceso a todas ellas, en alguna forma, es acceder al aprendizaje y a la ciudadanía porque el ciudadano de esta nueva sociedad necesita conocer el lenguaje de la política, de la economía, de la ciencia y la tecnología, del arte, de la literatura, de la filosofía...o, como se dice ahora en terminología educativa, necesita desarrollar todas estas competencias básicas.

Además de reconocer y valorar esta multiplicidad de voces, las nuevas prácticas letradas ya no son sólo actos académicos o lecturas silenciosas en bibliotecas; también son, cada vez más, actuaciones, puestas en escena, juegos, simulaciones, etc. En la línea bajtiniana, muchos de ellos llegan a parodiar o ridiculizar las formas culturales hegemónicas, por ejemplo el tatuaje, la escritura en el cuerpo o los grafitti son a primera vista usos "desviados" de las prácticas canónicas de escritura, que penalizaba, precisamente, el emborronar un libro o cualquier otra superficie no admitida.

Sea como fuere, el éxito de Internet no está en las herramientas en sí sino en la capacidad de apropiación social y cultural que han hecho de él los usuarios, gracias a que la red está en permanente construcción y renegociación, así como a su estructura abierta, ya que la red no tiene fronteras físicas, ni centro o periferia, ni arriba ni abajo, ni adentro ni afuera, ni pasado o futuro. Es decir, es capaz de sustituir el lenguaje lineal o secuencial del libro impreso por otra cosa distinta de la que son buenas muestras el hipertextos y los hipermedia.

En definitiva, lo importante no es la tecnología en sí, sino las prácticas de lectura y escritura que dan intención a los actos de creación de sentido. Tampoco debemos olvidar que estamos ante una cultura esencialmente híbrida: hay que integrar todos los artefactos culturales, cada cultura se conoce muy por sus artefactos. De ahí lo que hemos llamado este alcance expandido e integrador que dan estas nuevas corrientes teóricas y prácticas sociales (con especial énfasis en la cibercultura) al concepto clásico de "cultura escrita", que se ve así no limitado sino enriquecido y potenciado.

${ }^{22}$ GUTIÉRREZ, P., PUJOL, J. (2007) Propuestas para una difracción crítica del trabajo etnográfico. Vid. http://psicologiasocial.uab.es/fic/ca/node/126 


\section{Referencias bibliográficas}

Ames, P. (2002) Para ser iguales, para ser distintos. Educación, escritura y poder en el Perú. Lima: Instituto de Estudios Peruanos.

Aries, P. (1992). Historia de la Vida Privada. Taurus: España

Bakhtin, MM. (1986). Speech Genres and Other Late Essays. Austin: University of Texas Press.

Barré de Miniac, C. (2004). La littéracie. Conceptions théoriques et pratiques d'enseignement. L'Harmattan.

Bartoli Langeli, A. (1978). Intervento di apertura. Alfabetismo e Cultura Scritta nella storia della. società italiana, Atti del Seminario tenutosi a Perugia, 29-30 marzo 1977. Perugia: Università degli Studi

Barton, D y Tusting, K. (2005) Beyond Communities of Practice: Language, Power and Social Context. Londres: Cambridge University Press.

Barton, D. (1991). Literacy: An Introduction to the Ecology of Written Language. Oxford: Blackwell.

Barton, D. y Hamilton, M. (2000) Local Literacies. Reading and Writing in One Community. Londres: Routledge. Versión española del primer capítulo: "La literacidad entendida como práctica social", en Zavala, Niño-Murcia y Ames ed. (2004), 109-139.

Barton, D., Hamilton M. e Ivanic R. (2000). Situated literacies: reading and writing in context. Londres: Routlege.

Bordieu, P. (1983) Espacio social y campo de poder. Ed. Anagrama: Barcelona.

Bordieu, P. y Wacquant L.J.D. (1992) An Invitation to Reflexive Sociology. Chicago, Londres: Univ of Chicago Press.

Cassany, D. (2006) Tras las líneas. Sobre la lectura contemporánea. Anagrama.

Castillo Gómez, Antonio (2001a) (coord.): Historia de la cultura escrita. Del Próximo Oriente Antiguo a la sociedad informatiza, Gijón: Trea

Castillo Gómez, Antonio (2001b) (ed.): Cultura escrita y clases subalternas: una mirada española, Oiartzun: Sendoa

Castillo Gómez, Antonio A. y Sáex, C. (1994): Paleografía versus alfabetización. Reflexiones sobre historia social de la Cultura Escrita. Signo, Revista de Cultura Escrita, 1, 133-168

Chartier, R. (1994): El orden de los libros: lectores, autores, bibliotecas en Europa entre los siglos XIV y XVIII. 1992. Barcelona: Gedisa.

Chartier, R. (1982): «Intellectual History or Socio-Cultural History. The French Trajectories». En: Kaplan, S.L y La Capra, D. (eds.) Reappraisals in Modern European Intellectual History. Cornell University Press. Cavallo, G. y Chartier, R. (dirs.) (1998): Historia de la lectura en el mundo occidental. Madrid: Taurus.

Chartier, R. (1993): Libros, lecturas y lectores en la Edad Moderna, Madrid :Alianza Universidad. 
Chartier, R. y Roche, D. (1919). El libro. Un cambio de perspectiva. En Le Goff, J. y Nora, P. (dir.) Hacer la historia, III: Objetos nuevos. Barcelona: Laia

Cipolla, Carlo M. (1969). Literacy and Social Development in the West. Harmondsworth. Penguin (traducción española Educación y desarrollo en Occidente. Barcelona: Ariel, 1970).

Conti, Piero; Franchini, Giuliana; Gibelli, Antonio (dirs.) (2002): Storie di gente comune nell'Archivio Ligure della Scrittura Popolare. Génova: Universitá degli Studi di Genova-DISMEC, Acqui Terme EIG.

Dennett, D. (1995). La peligrosa idea de Darwin, Barcelona: Galaxia Gutenberg

Elias, N. (1978). The Civilizing Process. New York: Pantheon Books

Even-Zohar, I. (1990). Polysystem Studies. En Poetics Today, 11 (1)

Febvre, L. (1962). Pour une Histoire à part entière. Paris: Sevpen

Fleury M. y Valmary P. (1957). Les progrès de l'instruction élémentaire de Louis XIV à Napoleon III d'après l'enquête de Louis Maggiolo (1877-1879). Population, 12, 71-92.

Foucault, M. (2004). Vigilar y castigar: nacimiento de la prisión (“Surveiller et punir"), $1^{a}$ ed. Buenos Aires: Siglo XXI Editores Argentina

Gee, J. P. (1990). Social Linguistics and Literacies. Ideology in Discourses. Londres: Falmer Press.

Goody, J. y Watt, Ian. (1963). The consequences of literacy. En Comparative Studies in Society and History, 5, 304-345.

Goody, J. (1983). La domesticación del pensamiento salvaje. Madrid: Akal.

Graff, H. J. (1981). Literacy in History: An Interdisciplinary Research Bibliography. New York: Garland Publishing, Inc

Havelock, E.A (1996). La musa aprende a escribir. Reflexiones sobre oralidad y escritura desde la Antigüedad hasta el presente. Barcelona : Paidós.

Havelock, E.A. (1976): Origins of Western Literacy. Toronto: Ontario Institute for Studies in Education

Heath, S. (1983) Ways with words: Language, Life and Work in Communities and Classrooms. Cambridge: CUP.

Hoggart, R (1959). The Uses of Literacy. London: Chatto and Windus

Isnard, E. (2009). Jack Goody, Pouvoirs et savoirs de l'écrit. En Rev. L'Homme, 189. Lévi-Strauss et la cybernétique. Oralité et écriture. Nomades

Jenkins, H. (2008). Convergence Culture: La cultura de la convergencia en los medios de comunicación. Barcelona: Paidós

Lyons, M. (2007). Personal Narratives/Ordinary Writings. Towards a history of writing practices in the 19th and 20th centurie. Berna: Peter Lang,

Marchant Rivera, A. (1993): Literatura e historia de la cultura escrita: prácticas bibliófilas y escriturarias. En El Quijote de Cervantes. Málaga: Universidad de Málaga. 
Martos García, A. (2008). Bases para la elaboración de un sistema de información sobre la cultura escrita en el Quijote (Inédita - tesis doctoral Universidad de Extremadura)

Mullett, Michael A. (1987). Popular culture and popular protest in late medieval and early modern Europe. London and New York: Croom Helm

Oakeshott, M. (2009) La voz del aprendizaje liberal. Barcelona: Katz Editores.

Olson, D. (1994). El mundo sobre el papel. Barcelona : Gedisa

Petrucci, A. (1979). Funzione della scrittura e terminologia paleografica. En Palaeographica, diplomatica et archivistica. Studi in onore di Giulio Battelli, I. Roma: Edizioni di Storia e Letteratura, (Storia e Letteratura. Raccolta di studi e testi, 139).

--- (1993). Breve storia della scrittura latina, Roma: Il Bagatto

Viñao Frago, A. (1984). Del analfabetismo a la alfabetización. Análisis de una mutación antropológica e historiográfica. Historia de la Educación, 3, 151-189.

--- (1992). Alfabetización y alfabetizaciones. En Escolano, A. (ed.) Leer y escribir en España. Doscientos años de alfabetización (pp.385-386) Madrid: Fundación Germán Sánchez Ruipérez.

Rubalcaba Pérez, C (2006): Entre las calles vivas de las palabras. Prácticas de Cultura Escrita en el Siglo XIX. Gijón: Trea.

Ruiz, E. (1992). Hacia una semiología de la escritura. Madrid: Pirámide.

Sáez Sánchez, C. y Castillo Gómez, A. (1994). Paleografía versus alfabetización. Reflexiones sobre historia social de la Cultura Escrita. Signo. Revista de Historia de la Cultura Escrita, 1, 133-168

Schofield, R. (1968). The measurement of literacy in pre-industrial England. En Goody, J. (ed). . Literacy in Traditional Societies. New York: Cambridge University Press

Schuliaquer, I. Entrevista a Daniel Link en Revista El Monitor, no 3. Consultado el 22 de Abril de 2010, disponible en línea en http://www.mcye.gov.ar/monitor/nro3/dossier7.htm

Sierra Blas, Verónica (2003). Aprender a escribir cartas. Los manuales epistolares en la España contemporánea (1927-1945). Gijón: Trea

Street, B. (1984). Literacy in theory and practice. Nueva York: Cambridge University Press.

Stone, L. (1964). The Educational Revolution in England, 1560-1640. Past and Present, XXVIII, 1, 41-80

Tamara Garay, A. (2005). El entramado de la oralidad en El queso y los gusanos: La historia de Carlo Ginzburg. En La casa de Asterión. Revista Trimestral de Estudios Literarios. 22 (VI). Disponible en línea en http://casadeasterion.homestead.com/v6n22ginz.html

Vincent, D. (1989). Literacy and Popular Culture, England 1750-1914. Cambridge: Cambridge University Press.

Vivas Moreno, A. (1998). Fondos documentales del Archivo Histórico de la Universidad de Salamanca. La Colección de Papeles Varios: Análisis 
descriptivo, tesauro y gestión documental automatizada. Tesis doctoral. Salamanca, 6 vols.

Wenger, Etienne (2001). Comunidades de práctica: aprendizaje, significado $e$ identidad. Barxcelona: Paidós.

Zavala, V. (2002). (Des)encuentros con la escritura. Escuela y comunidad en los andes peruanos. Lima: Red para el desarrollo de las ciencias sociales en el Perú.

(Artículo recibido: 22-09-2010; revisado: 22-10-2010; aceptado: 19-11-2010) 